\title{
Overexpression of Raf-1 kinase inhibitor protein inhibits cell invasion and migration in lung cancer cells through suppressing epithelial-mesenchymal transition
}

\author{
Qin Wang ${ }^{1 \#}$, Xin-Ying Li ${ }^{1,2 \#}$, Bing Wan ${ }^{3 \#}$, Jie Zhang ${ }^{1}$, Rong Sun ${ }^{1}$, Chu-Yao Zhou ${ }^{1}$, Ping Zhan ${ }^{1,2}$, \\ Yong Song ${ }^{1,2}$ \\ ${ }^{1}$ Department of Respiratory and Critical Care Medicine, Jinling Hospital, Nanjing University School of Medicine, Nanjing 210002 , China; ${ }^{2}$ Nanjing \\ University Institute of Respiratory Medicine, Nanjing 210023, China; ${ }^{3}$ Department of Respiratory and Critical Care Medicine, The Affiliated \\ Jiangning Hospital of Nanjing Medical University, Nanjing 211100, China \\ Contributions: (I) Conception and design: Q Wang; (II) Administrative support: P Zhan, Y Song; (III) Provision of study materials or patients: J \\ Zhang, R Sun, CY Zhou;(IV) Collection and assembly of data: XY Li, B Wan; (V) Data analysis and interpretation: Q Wang, XY Li; (VI) Manuscript \\ writing: All authors; (VII) Final approval of manuscript: All authors. \\ \#These authors contributed equally to this work. \\ Correspondence to: Ping Zhan; Yong Song. Department of Respiratory and Critical Care Medicine, Jinling Hospital, Nanjing University School of \\ Medicine, Nanjing University Institute of Respiratory Medicine, 305 East Zhongshan Road, Nanjing 210002, China. Email: zhanping207@163.com; \\ yong_song6310@yahoo.com.
}

Background: Raf-1 kinase inhibitor protein (RKIP) is a small evolutionary conserved protein that was associated with the Raf-MEK-ERK pathway. However, whether RKIP would alter the invasion and metastasis of non-small cell lung cancer (NSCLC) and play the role through suppressing epithelialmesenchymal transition (EMT) remains to be explored.

Methods: A549 cells were transfected with RKIP-GV141 plasmid for overexpression of RKIP. Colony formation assay and MTT assay were performed to measure the effects of RKIP on the proliferation and cell viability assay of A549 cells. Transwell, Migration Assay and wound healing assay were performed to analyze the effects of RKIP on the invasion and metastasis of A549 cells. E-cadherin and vimentin were measured by Western blot to conform RKIP affects invasion and metastasis of NSCLC via inhibiting EMT.

Results: RKIP is downregulated in NSCLC tissues compared to adjacent normal lung tissues by IHC. Decreased expression of RKIP contributes to poor prognosis in lung adenocarcinoma patients. Age and pTNM stage were independent prognostic factors for adenocarcinoma patients. Overexpression of RKIP reduces the cell viability and limits the proliferation, invasion, and migration of A549 cells in vitro. Wound healing assay showed the degressive ability of metastasis. High expression of E-cadherin and low expression of vimentin indicated that RKIP affects invasion and metastasis of NSCLC via inhibiting EMT.

Conclusions: RKIP is decreased in NSCLC tissues. Overexpression of RKIP in A549 cells would decrease the cellular proliferation, viability, invasion ability, and metastasis ability probably via inhibiting EMT through upregulating E-cadherin expression and downregulating vimentin expression.

Keywords: Raf-1 kinase inhibitor protein (RKIP); non-small cell lung cancer (NSCLC); proliferation; invasion; migration; metastasis; epithelial-mesenchymal transition (EMT)

Submitted Jul 04, 2019. Accepted for publication Sep 23, 2019.

doi: $10.21037 /$ tcr.2019.09.56

View this article at: http://dx.doi.org/10.21037/tcr.2019.09.56 


\section{Introduction}

According to the report produced by the International Agency for Research on Cancer in 2018 (1), lung cancer is still the most common cancer and the main reason of cancer related death, which accounts for $11.6 \%$ of all identified cases and $18.4 \%$ of all related deaths regardless of genders. Despite rapid advances in diagnostic and therapeutic techniques for lung cancer, alive $\geq 5$ years after diagnosis of lung cancer patients is only $17.7 \%$ (2). Non-small cell lung cancer (NSCLC) accounted for more than $80 \%$. Although the increasing use of lung cancer screening may alter the most typical stages of diagnosis, most patients with NSCLC are diagnosed with advanced cancer.

Tumor metastasis has been regarded as a marker of the progression of cancer to advanced stage, but tumor cells may release from the primary lesion at an early stage and form distant micro-metastasis, which has been confirmed in the marrow cavity of early breast cancer (3). Cancer cells are separated from primary tumors by the action of cell adhesion molecules, which include cadherin, integrin, selectin and immunoglobulin superfamily. E-cadherin plays an important role. E-cadherin acts as a standard protein secreted by epithelial cells and plays a direct role in cellcell adhesion (4). It regulates the activity of EGFR through receptor tyrosine kinase, thereby mediating intracellular signal transduction. $C D H 1$, an E-cadherin gene, is an invasive suppressor gene. Its expression in NSCLC is related to prognosis. The decreased expression level of E-cadherin is related to the occurrence of EMT, while the expression level of vimentin is increased (5). There is no denying that EMT is of importance in the metastasis and invasion of tumors. Intensive research on signaling pathways and cytokine regulation is needed.

Raf-1 kinase inhibitor protein (RKIP), is a protein, which is part of the phosphatidylethanolamine protein (PEBP) family (6). It was recognized in 1999 that its function is mainly to inhibit Raf-1/MEK/ERK, NF- $\mathrm{BB}$ and survival signaling pathway. Studies in mouse prostate cancer models have shown that RKIP inhibits tumor metastasis (7). Prior investigations have corroborated that RKIP can govern the metastasis of various tumors such as gastric cancer (8), breast cancer (9), lung cancer and cervical cancer (10).

Previous studies in our team have found that RKIP expression decline in lung cancer tissues is related to lymph node metastasis and late pathological stage of cancer. It suggests that RKIP inhibits invasion and metastasis of cancer in NSCLC, and that RKIP expression is related to prognosis in adenocarcinoma, suggesting that RKIP inhibits metastasis of cancer directly affects prognosis. This study intends to establish a lung cancer cell model with high expression of RKIP in vitro, to investigate whether RKIP can inhibit the invasion of lung cancer in vitro and control its metastasis. Then we try to unearth whether the mechanism of inhibition is related to EMT.

\section{Methods}

\section{Patients and tissue specimens}

The study included 126 lung cancer patients who underwent surgery at the Nanjing Chest Hospital and Jinling Hospital Affiliated to Nanjing University School of Medicine from May 2007 to May 2009. Postoperative pathological grading was reviewed in the light of TNM staging (7th edition). In addition, 75 specimens of lung adenocarcinoma and paracancerous normal tissues confirmed by surgery and pathology from July 2004 to November 2007 were collected, and follow-up time was more than 5 years. Once collected, the specimens were stored in liquid nitrogen at $-80{ }^{\circ} \mathrm{C}$ until they were removed.

\section{Immune-histochemical (IHC) and scoring in tissue microarrays (TMAs)}

RKIP expression in tumor tissues and paired adjacent normal tissues from 75 patients with lung adenocarcinoma was detected using commercial TMAs produced by Shanghai Outdo Biotech, China. Antigen was retrieved by citrate buffer ( $\mathrm{PH} \mathrm{6.0)}$ with high pressure for $5 \mathrm{~min}$. Then we employed anti-RPIP antibody (Sigma-Aldrich) to incubate the sections overnight at $4{ }^{\circ} \mathrm{C}$. The negative control group replaced the original antibody with PBS. The clinical data were then blindly examined by two pathologists independently. The staining fraction of protein expression was described in detail before. The overall protein score varies from 0 to 12 , depending on the product of intensity and positive score.

\section{Cell lines and culture conditions}

The human NSCLC cell line A549 was obtained from the American Type Culture Collection (CCL-185), and cultured in Dulbecco's modified Eagle's medium containing $10 \%$ FBS (Gibco BRL) as recommended. 


\section{Plasmids, antibodies and reagents}

The human RKIP expressing vector is created by inserting the full length CDS of RKIP gene ((NM_002567)) into GV141 vector (Genechem, Shanghai, China) digested with $\mathrm{XhoI}$ and $\mathrm{KpnI}$ in the sense orientation. The empty vector was used as a control in this study. All plasmids were confirmed by sequencing analysis. RKIP (\#13006), Vimentin (\#3932) and E-cadherin (\#3195) antibodies were from Cell Signaling Technology, and beta-Actin antibodies (A5316) were purchased from Sigma-Aldrich. Lipofectamine 2000 and G418 (Geneticin) was purchased from Life Technologies. Dimethyl sulfoxide (DMSO) and MTT [3, (4,5-dimethylthiazol-2-yl)-2, 5-diphenyltetrazolium bromide] were also bought in Sigma-Aldrich. DNA Transfection

Cells were transfected with plasmids using Lipofectamine 2000 reagent on the basis of the manufacturer's instructions. In brief, around $15 \times 10^{4}$ cells were plated in 6 -well plate 24 hours prior to transfection. The $2 \mu \mathrm{g}$ of plasmids DNA and $5 \mu \mathrm{L}$ of transfection reagent were resuspended in equal amount of no-serum medium, respectively. After $5 \mathrm{~min}$ of incubation, mixed the DNA and reagent solution well and kept at room temperature for $20 \mathrm{~min}$, add the complexes drop wisely into each well containing $2 \mathrm{~mL}$ culturing medium without antibiotics. Continue culturing the cells for 72 to 96 hours and harvest cells for subsequent experiments.

\section{Extraction of $R N A$, reverse transcription, and real-time PCR}

Total RNAs were extracted from A549 cells at Day 3 posttransfection using Trizol Reagent (Invitrogen) following the instructions. The remaining genomic DNA was digested with DNase I. The primeScriptTM RT Master Mix kit (TaKaRa) was employed to synthesize the DNA. The cDNA samples were diluted 2-fold and subjected to realtime PCR assays utilizing the $\mathrm{SYBR}^{\circledR}$ Premix Ex TaqTM II kit (TaKaRa). The PCR experiments were carried out with ABI PRISM 7500 Fast Real-Time PCR System by sequential incubation for $2 \mathrm{~min}$ at $50^{\circ} \mathrm{C}, 2 \mathrm{~min}$ at $95^{\circ} \mathrm{C}$, 40 cycles at $95^{\circ} \mathrm{C}$ for 15 seconds, and incubated at $60^{\circ} \mathrm{C}$ for 30 seconds. Primers for real-time RT-PCR experiments: $\beta$-Actin, Forward 5'-CTCTTCCAGCCTTCCTTCCT-3', Reverse 5'-AGCACTGTGTTGGCGTACAG-3', RKIP, Forward 5'-AGTTCAGTGTTGCATGTATAATAG-3', Reverse 5'-GAGTGAGTGAGTAAAATTATAAAGG-3'.

\section{Western blot}

After 4 days of transfection, A549 cells were collected and lysed with RIPA buffer supplemented with $1 \mathrm{mM}$ PMSF (Roche). Protein concentrations were measured by BioRad Protein Assay Kit (BioRad) with bovine serum albumin (Sigma) as the standard. Then $40 \mu \mathrm{g}$ protein samples were mixed with $5 \times$ SDS loading buffer $(0.01 \%$ bromophenol blue, 7\% DTT, 10\% SDS, 50\% glycerol, $50 \mathrm{mM}$ Tris $\mathrm{pH}$ 6.8) and denatured by heating at $95{ }^{\circ} \mathrm{C}$ for 5 minutes and cooled on ice. The protein sample was resolved on a $4 \%$ stacking/10\% resolving polyacrylamide gel (SDS-PAGE) at $120 \mathrm{~V}$ for around 1.5 hours. After electrophoresis, the protein was transferred to a PVDF membrane at $100 \mathrm{~V}$ for 3.0 hours. $5 \%$ skim milk prepared with TBST solution ( $1 \times$ TBS, $0.1 \%$ Tween-20) was intended to block the PVDF membrane for 2 hours on a slow shaker at room temperature. The membrane was immunoblotted with antibodies as indicated at $4{ }^{\circ} \mathrm{C}$ overnight. Eventually, the membrane was soaked with ECL luminescent solution (Thermo Fisher Scientific) for Western blotting.

\section{Colony formation assay}

The colony formation assay was carried out to determine the proliferative capability of A549 cells. Briefly, cells transfected with indicated plasmids were replanted into new 6-well plates at 2,000 cells per well and incubated for 14 days in selection medium containing $500 \mu \mathrm{g} / \mathrm{mL}$ of G418. Colonies were immobilized with $70 \%$ ethanol for 15 minutes, stained with $0.5 \%$ crystal violet for 20 minutes, and stained continuously for $20 \mathrm{~min}$ at room temperature. The soft agar colony formation assay is performed to monitor the cells' anchorage-independent growth. Cells were mixed in $0.35 \%$ agarose and layered on top of the base layer of $0.5 \%$ agarose. Continue culturing for 4 weeks and stain plates with $0.005 \%$ crystal violet.

\section{Cell viability assay}

A549 cells transfected with indicated plasmids were replanted in 6-well plates in triplicate. After stained by trypan blue dye, the cells were counted on the hemacytometer chamber. The MTT assay was also used to determine cell viability. Briefly, the cells transfected with indicated plasmids were seeded in a 96-well plate and cultured for 2-4 days. Added $1 / 10$ volume of $5 \mathrm{mg} / \mathrm{mL}$ 
MTT to the medium and incubated for $4 \mathrm{~h}$ at $37^{\circ} \mathrm{C}$. MTT was dislodged from each well and the appropriate amount of DMSO dissolved the cells. The dark blue Formosa crystals formed in intact cells were shaken on a shaker for $15 \mathrm{~min}$ to dissolve. The results obtained at $570 \mathrm{~nm}$ was the absorbance of the Elx800 ELISA analyzer microplate reader (BioTek Instruments, Inc., Winooski, VT, USA).

\section{Migration and invasion assays}

The cell motility and cell invasive abilities were appraised by way of Transwell (Corning Life Sciences) and Matrigel invasion (BD Biosciences), respectively. For Transwell migration assay, A549 cells transfected with indicated plasmids were split into the upper compartment of the chamber at $10 \times 10^{4}$ per well. Whereas $15 \times 10^{4}$ cells per well were seeded for the invasion assay. For both migration and invasion assay, cells were cultured in medium containing only $1 \%$ FBS in the upper compartment of the chamber, while a conventional medium containing $10 \%$ fetal calf serum was added to the lower chamber. Twenty-four hours later, the cells at the top of the membrane were scraped off with a cotton swab, and the cells that migrated to the bottom were fixed with methanol and stained with $0.5 \%$ crystal violet. Stained cells were counted under a microscope at a magnification of 200x. For each replicate, the tumor cells in 6 randomly selected fields were determined. The average number of migrated or invaded cells was expressed as percentage relative to control cells. Each experiment needs to be repeated 3 times and the average is calculated.

\section{In vitro scratch wound healing assay}

A549 cells transfected with indicated plasmids were seeded onto 6-well plates in a density that, after 24 hours of growth, they should reach $70-80 \%$ confluence as monolayer. Scrape the single layer gently and slowly with the tip of a new $200 \mathrm{~mL}$ pipette through the center of the hole (Do not change the medium). After gently rinsing detached cells off with PBS, cells were incubated in medium containing $1 \%$ FBS at $37{ }^{\circ} \mathrm{C}$ in a humidified $5 \% \mathrm{CO}_{2}$ incubator. Images were taken after $30 \mathrm{~h}$ and the subsequent re-colonization of stripped surface was quantified by measuring the distance between the wound edges at three randomly selected fields. The percentage of wound healing was determined as the area of the wound remaining after $30 \mathrm{~h}$ normalised against the initial area of the wound (0 h) using ImageJ.

\section{Results}

\section{RKIP is downregulated in NSCLC tissues}

Previously, our group showed that the expression level of RKIP mRNA in cancer tissues was noticeably lower than that in normal adjacent tissues. An obvious relativity existed between low RKIP mRNA expression and the higher N-stage of pathological TNM stage (11). In order to determine the expression profile of RKIP in NSCLC, we collected 75 matched clinical NSCLC tissues and adjacent normal tissues.

The expression of RKIP protein in clinical specimens was detected by IHC method (Figure 1). The results show that RKIP in normal alveolar tissue adjacent to carcinoma cell nucleus has different degree of expression while no expression in the cytoplasm. There were obvious differences in the expression of RKIP in cancer tissues. Some RKIP was only expressed in the nucleus, some were expressed in the nucleus and cytoplasm, and some were negative, according to the differences in the expression of RKIP in tumor tissues and adjacent tissues. The positive value of RKIP is positive for cytoplasmic expression.

\section{Decreased expression of RKIP leads to poor prognosis of lung adenocarcinoma patients}

We assessed the correlation between RKIP reduction and clinicopathological factors of lung adenocarcinoma patients. In the previous study (11), 126 patients were divided into the RKIP mRNA high expression group and the low expression group, and it was found that the down-regulation of RKIP expression level was correlated with lymph node metastasis $(\mathrm{P}=0.019)$ and late pTNM stage $(\mathrm{P}=0.015)$. However, it was not related to clinicopathological features such as gender, age, histological type, and primary tumor size $(\mathrm{P}>0.05)$. Wang et al. (12) observed reduced RKIP protein expression levels in patients with late-stage NSCLC $(\mathrm{P}=0.008)$ and $57.32 \%$ patients with metastasis exhibited low RKIP expression levels $(\mathrm{P}=0.001)$. Xie et al. (13) indicated that not only clinical $\mathrm{T}$ stage bot also RKIP expression level had a huge influence on the overall survival of NSCLC patients. Whereas, in this study, for patients with lung adenocarcinoma who were followed up for 5 years, the results showed that the expression level of RKIP in lung adenocarcinoma was not significantly correlated with the clinicopathologic features (Table 1). As for the prognosis, patients with higher levels of RKIP protein had 
Lung adenocarcinoma tissues
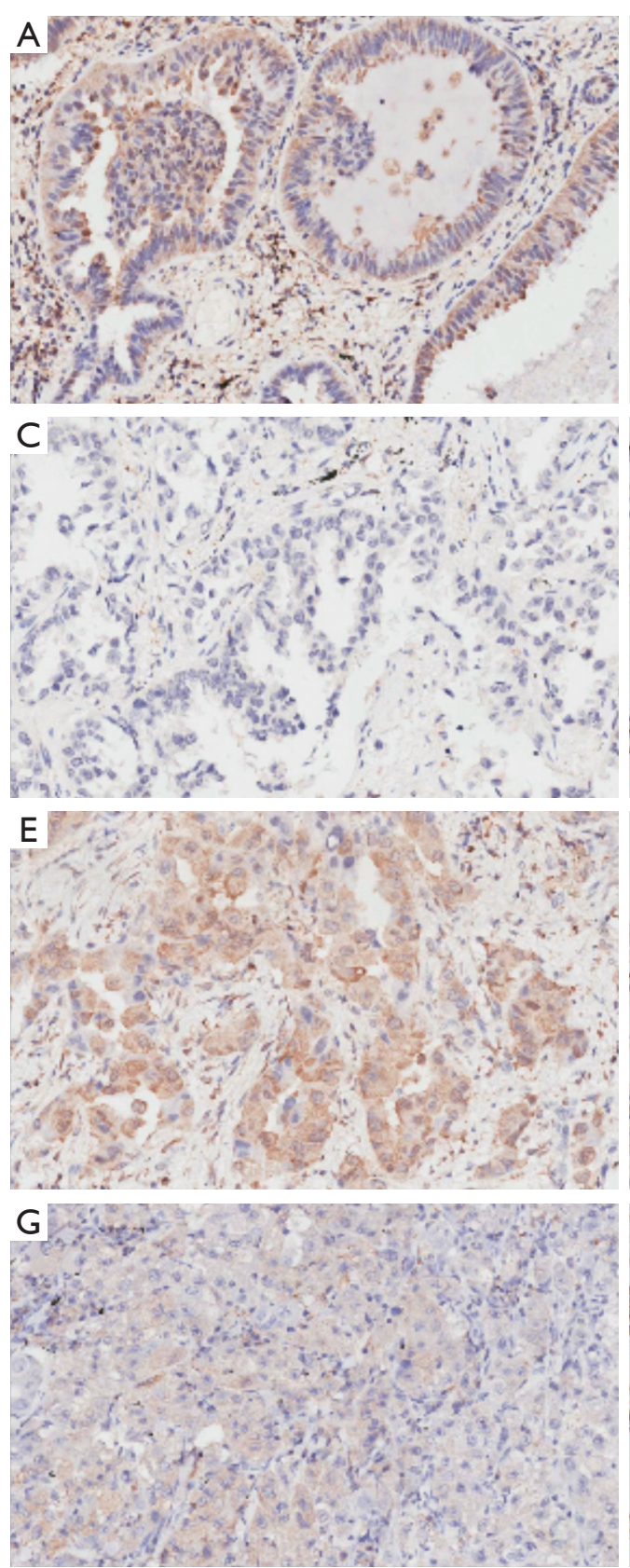

Paired adjacent non-tumoral tissues
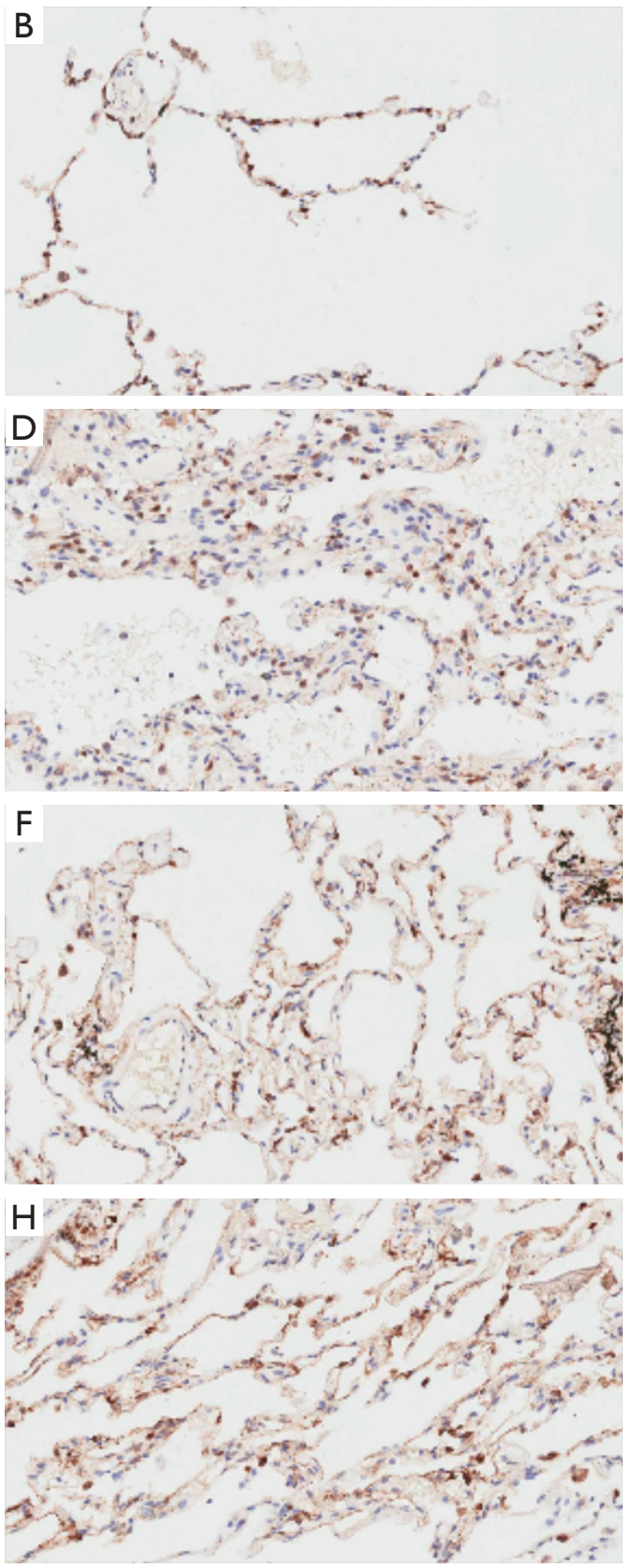

Figure 1 RKIP is downregulated in NSCLC tissues. IHC investigation of RKIP protein expression levels in lung adenocarcinoma (AD) tissues and paired adjacent non-tumoral tissues ( $\mathrm{n}=75$ ). (A) RKIP was positively expressed in the cytoplasm and nucleus of alveolar cell carcinoma, mainly cytoplasm; (B) partial expression of RKIP in the nuclei of corresponding paracancerous alveolar tissues; (C) negative expression of RKIP in lung adenocarcinoma; (D) partial expression of RKIP in the nuclei of corresponding paracancerous tissues; (E) positive expression of RKIP in lung adenocarcinoma was observed in both cytoplasm and nucleus, mainly cytoplasm; (F) partial expression of RKIP in the nucleus of the corresponding paracancerous tissues; $(G)$ positive expression of RKIP in lung adenocarcinoma was only expressed in the cytoplasm; (H) partial expression of RKIP in the nucleus of corresponding cancer tissues. Original magnification, $\times 40$. RKIP, Raf-1 kinase inhibitor protein; NSCLC, non-small cell lung cancer; IHC, immune-histochemical. 
Table 1 RKIP protein expression and the association with the clinicopathological factors in 75 lung adenocarcinomas

\begin{tabular}{|c|c|c|c|c|}
\hline \multirow{2}{*}{$\begin{array}{l}\text { Clinical } \\
\text { characteristics }\end{array}$} & \multirow{2}{*}{$\begin{array}{c}\text { Number of } \\
\text { patients (\%) }\end{array}$} & \multicolumn{3}{|c|}{ RKIP protein expression } \\
\hline & & Low & High & $P$ \\
\hline All patients & 75 & 14 & 61 & \\
\hline \multicolumn{2}{|l|}{ Gender } & & & 0.109 \\
\hline Male & $39(52.0)$ & 10 & 29 & \\
\hline Female & $36(48.0)$ & 4 & 32 & \\
\hline \multicolumn{2}{|l|}{ Age } & & & 0.050 \\
\hline$<65$ у. о. & $47(62.7)$ & 12 & 35 & \\
\hline$\geq 65$ у. о. & $28(37.3)$ & 2 & 26 & \\
\hline \multicolumn{2}{|l|}{ Size of tumor } & & & 0.671 \\
\hline$\leq 3 \mathrm{~cm}$ & $36(48.0)$ & 6 & 30 & \\
\hline$>3 \mathrm{~cm}$ & $39(52.0)$ & 8 & 31 & \\
\hline \multicolumn{2}{|c|}{ Pathological grade } & & & 0.050 \\
\hline I & $14(18.7)$ & 1 & 13 & \\
\hline II & $39(52.0)$ & 6 & 33 & \\
\hline III & $22(29.3)$ & 7 & 15 & \\
\hline \multicolumn{3}{|c|}{ Lymph node metastasis (pN) } & & 0.387 \\
\hline No & $40(53.3)$ & 6 & 34 & \\
\hline N1-3 & $35(46.7)$ & 8 & 27 & \\
\hline \multicolumn{2}{|l|}{ p-TNM stages } & & & 0.216 \\
\hline I & $36(48.0)$ & 5 & 31 & \\
\hline II & $17(22.7)$ & 3 & 14 & \\
\hline III & $19(25.3)$ & 5 & 14 & \\
\hline IV & $3(0.04)$ & 1 & 2 & \\
\hline
\end{tabular}

RKIP, Raf-1 kinase inhibitor protein.

a longer survival time $(\mathrm{P}=0.028, \log$-rank test, Figure 2). In addition, multivariate Cox regression analysis revealed Age ( $\mathrm{HR}=3.398,95 \% \mathrm{CI}: 1.532-7.538, \mathrm{P}=0.030)$ and pTNM stage $(\mathrm{HR}=2.016,95 \% \mathrm{CI}: 1.144-3.552, \mathrm{P}=0.015)$ were independent prognostic factors for adenocarcinoma patients (Table 2).

\section{Overexpression of RKIP reduces the cell viability of NSCLC cells}

Firstly, the cell model with overexpression of RKIP was constructed by transfecting RKIP-containing plasmid into A549 cells. The transfection efficiency was verified by

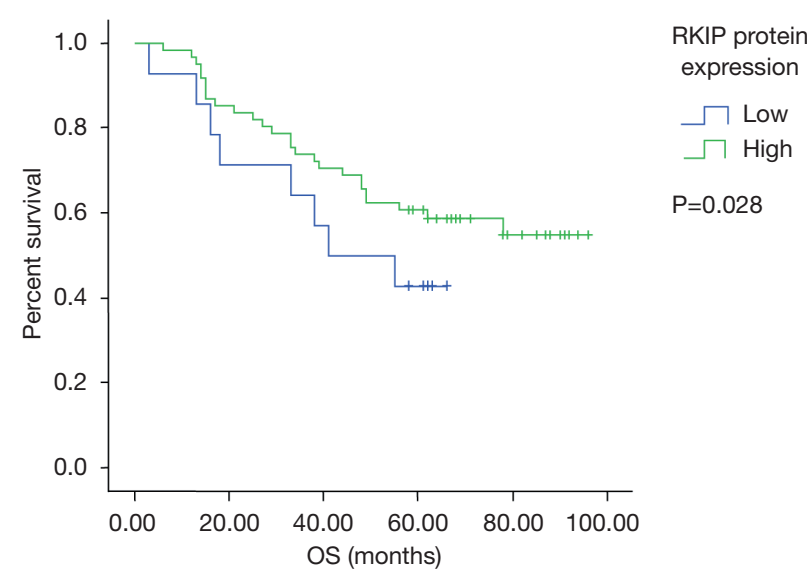

Figure 2 K-M survival curve shows the effect of RKIP expression on the survival of patients with lung adenocarcinoma. RKIP, Raf-1 kinase inhibitor protein.

Table 2 Multivariate Cox analysis showed the effect of each factor on prognosis

\begin{tabular}{lcc}
\hline \multirow{2}{*}{ Factors } & \multicolumn{2}{c}{ OS } \\
\cline { 2 - 3 } & HR $(95 \% \mathrm{Cl})$ & $\mathrm{P}$ \\
\hline RKIP (high/low) & $0.583(0.369-1.828)$ & 0.629 \\
Age (>65/<65 years) & $3.398(1.532-7.538)$ & 0.030 \\
Gender (female/male) & $0.821(0.474-1.715)$ & 0.752 \\
Size of tumor (>3/<3 cm) & $0.794(0.348-1.814)$ & 0.585 \\
Pathological grade (poor/ & $0.939(0.502-1.756)$ & 0.844 \\
medium-high differentiation) & & \\
N-stage (N1-3/N0) & $1.526(0.654-3.564)$ & 0.329 \\
pTNM stage (III-IV/I-II) & $2.016(1.144-3.552)$ & 0.015 \\
\hline
\end{tabular}

RKIP, Raf-1 kinase inhibitor protein.

RT-PCR and Western blot at mRNA and protein levels (Figure 3A,B). Current research suggests that RKIP as a tumor suppressor gene, it plays its role through inhibiting tumor invasion and metastasis, but has no obvious effect on primary tumors. The cell viability of the RKIP high expression group was significantly higher than that of the control group (Figure 3C,D). Flat-plate cloning experiments can directly reflect the proliferation of cells by calculating the clone formation rate. Soft AGAR cloning experiments can also reflect the malignancy of tumors to a certain extent. The number and size of colonies of A549 cells were inhibited by ectopic expression of RKIP. Collectively, these data revealed that RKIP high expression can inhibit the 


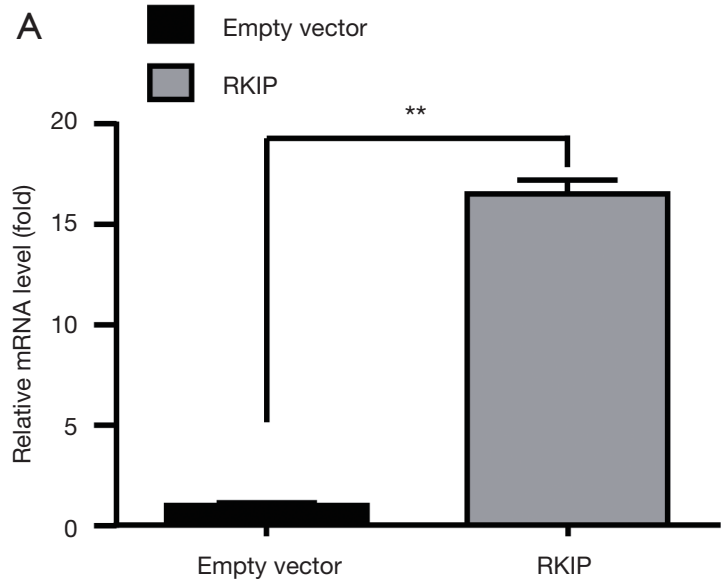

C

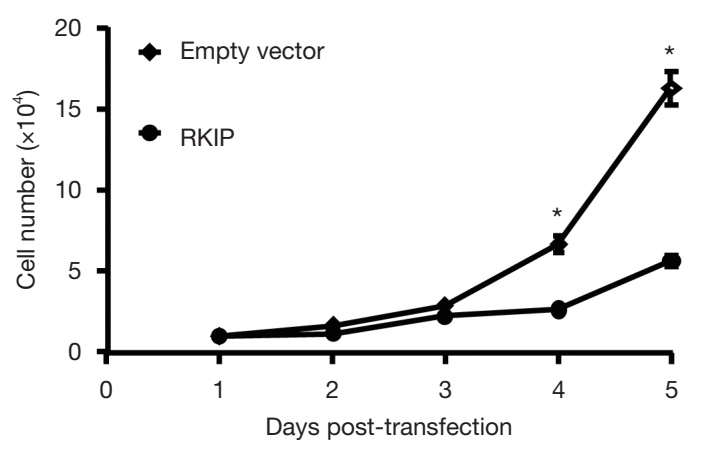

D

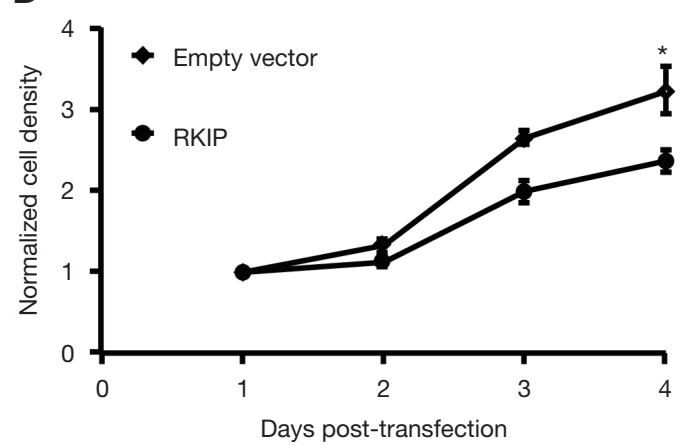

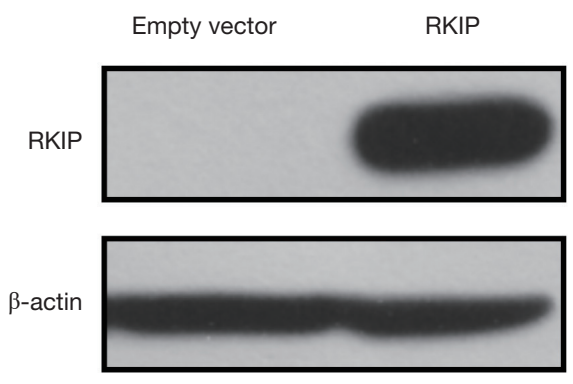

E
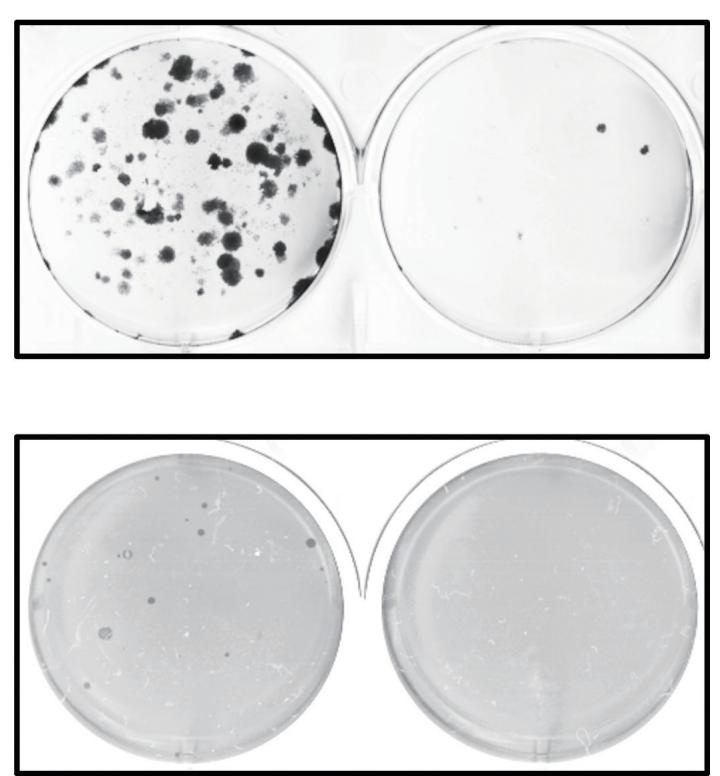

Figure 3 Overexpression of RKIP reduced the cell viability of A549. (A) RT-qPCR indicated that the mRNA expression level of RKIP was significantly increased after transfection; (B) western blot indicated that the protein expression level of RKIP was significantly increased after transfection; (C) tryptophan blue stain test and (D) MTT test indicated that the cell viability of the group with high expression of RKIP was higher than that of the control group. RKIP, Raf-1 kinase inhibitor protein; (E) colony formation and Soft agar colony formation assay of A549 cells transduced with RKIP expressing plasmid or empty vector. ${ }^{*}, \mathrm{P}<0.05$; ${ }^{* *}, \mathrm{P}<0.01$. RKIP, Raf-1 kinase inhibitor protein. 
lung adenocarcinoma cell proliferation and cell vitality (Figure 3C,D,E).

\section{Overexpression of RKIP suppresses the invasion and migration of NSCLC cells}

Increased motility and enhanced migration of tumor cells are important features of tumor metastasis. Studies in many tumors, suggest that RKIP is associated with tumor migration. This study used RKIP plasmid transfection A549 cell scratch after the experiment, at 0 and 30 hours. The arrow was the edge of the scratch. The shortest distance of the scratch edge was measured by image $\mathrm{J}$ software (three different regions were measured at the same scratch site, and the average value was taken). The relative scratch width was used to reflect the migration ability of lung adenocarcinoma cells (Figure $4 A, B$ ). Transwell results demonstrated that high expression of RKIP significantly inhibited the invasion and metastasis of A549 cells (Figure 4C).

\section{Overexpression of RKIP inbibits EMT in NSCLC cells}

Although RKIP is currently recognized as a tumor metastatic suppressor gene, most studies about its mechanism of tumor metastasis are focused on the signaling pathway. RAF/ERK/STAT3 pathway (12) was considered to be a major signaling pathway associated with tumor metastasis.

EMT is an important step of tumor metastasis. Studies in prostate cancer cell lines have reported that RKIP inhibits tumor metastasis and may be related to inhibition of EMT (14). Our team found that the mechanism of RKIP inhibiting tumor metastasis was related to EMT. After transfection with RKIP plasmid, the expression level of RKIP protein was significantly increased compared with that of the empty vector group, and the expression level of e-cadherin, a representative marker of epithelial function, was significantly increased after transfection with RKIP, while the expression level of vimentin, a representative marker of mesenchymal cells, was significantly decreased, suggesting that high expression of RKIP inhibits epithelial-mesenchymal transformation (Figure 5). These data added further evidence that the inhibition of tumor invasion and metastasis by RKIP might be related to EMT at the nucleic acid level.

\section{Discussion}

Although many primary tumors can be controlled by surgery, chemotherapy and radiotherapy, once metastasis occurs, the prognosis of the tumor is very poor. Metastasis of the tumor extremely increases its mortality, especially in lung cancer. About $90 \%$ of lung cancer deaths are related to metastasis. Consequently, the molecular mechanism of invasion and metastasis of NSCLC urgently requires familiarity and understanding. We hope to find measures for the intervention of tumor invasion and metastasis and provide individualized treatment of NSCLC, so as to improve the prognosis of NSCLC patients.

In 1999, Yeung et al. (6) found that PEBP-1, as a Raf-1 binding protein, inhibited the phosphorylation and activation of MEK, renamed RKIP. PEBP-1/RKIP is initially clarified as a key factor in cell signaling cascade. Early studies have found that RKIP plays different roles in different species (15), organs (16) and cells (17). The expression of RKIP was detected in almost all mammalian tissues, mainly in the cytoplasm and the medial surface of the cell membrane (18). RKIP is a very important cell signaling factor, which can also antagonize the host immune response and promote the release of immune-related proteins (19).

RKIP is associated with a variety of tumors. The expression of RKIP in non-metastatic cell lines was found to be significantly higher in prostate cancer cells through gene chip analysis initially (6). Previous studies in vitro have shown that silencing RKIP expression increases the invasiveness of tumor cells, whereas restoring RKIP expression inhibits the invasiveness of tumor cells. Further experiments in vivo showed that the lung metastasis of prostate cancer cells with high expression of RKIP was significantly reduced, but the growth rate of primary tumor was not affected, suggesting that RKIP is a very important tumor metastasis suppressor gene. Herein, we disclosed that RKIP had lower mRNA and protein expression in lung adenocarcinoma tissues than in adjacent normal tissues. We further investigated the effect of reduced RKIP expression on poor prognosis in patients with lung adenocarcinoma.

In order to further verify the effect of RKIP on proliferation and viability of lung cancer cells, we established a lung adenocarcinoma cell model with high expression of RKIP in vitro by plasmid transfection of A549 cells. Clone formation and cell viability tests confirmed that high expression of RKIP inhibited the proliferation of A549 cells, and the cell viability also decreased significantly, suggesting that RKIP inhibited tumor growth. The occurrence and development of primary tumors were not correlated with RKIP, and the same conclusion was reached in other 
A

Empty vector
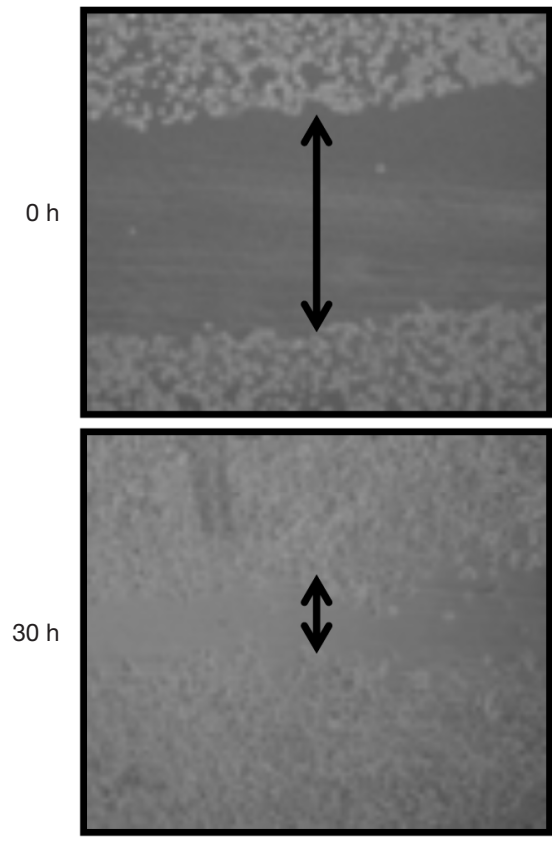

C

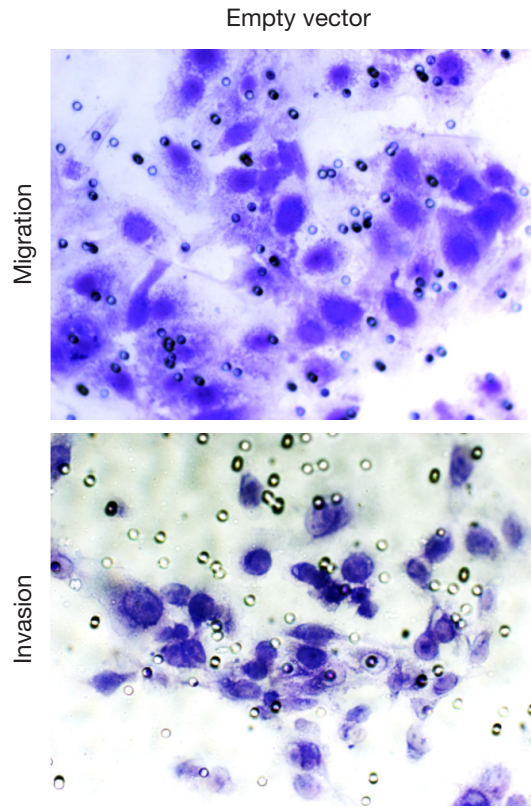

RKIP
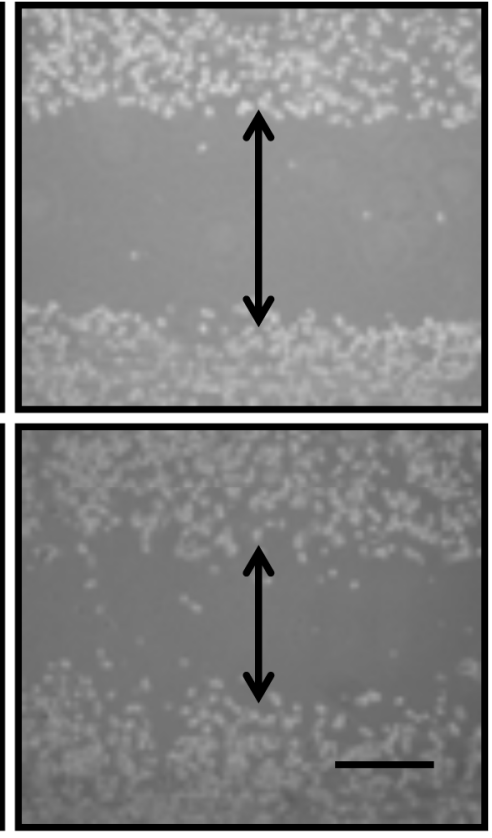

RKIP

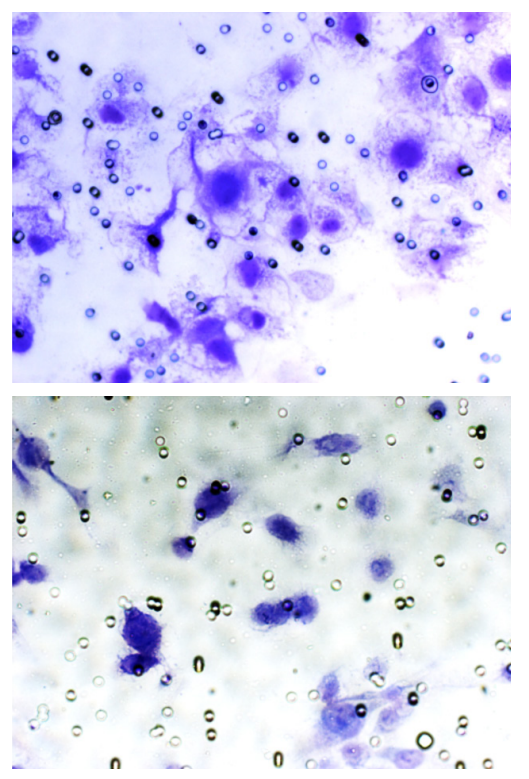

B
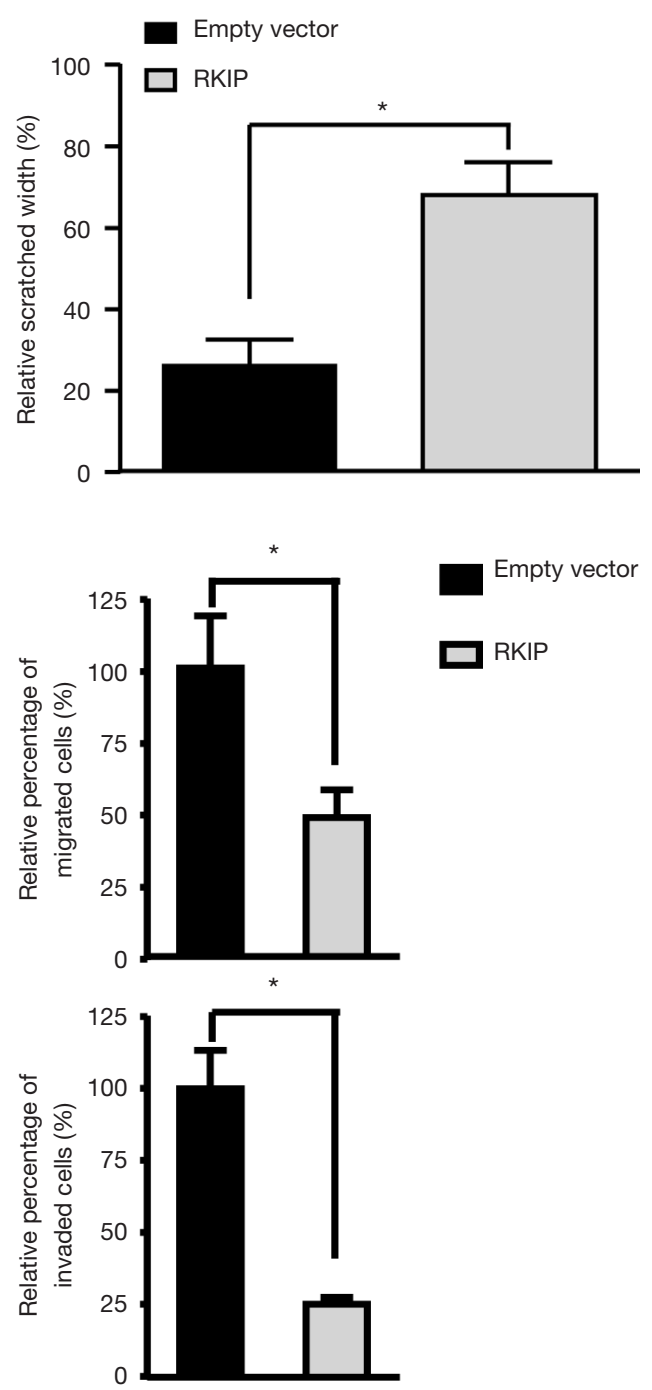

Figure 4 Overexpression of RKIP suppresses the invasion and migration of NSCLC cells. (A) Cell migration capacity of RKIP transfection significantly decreased after 30 hours of scratch test; (B) compared with the control group, the relative scratch width of A549 cells transfected with RKIP plasmid decreased significantly. *, $\mathrm{P}<0.05$; (C) transwell migration experiment: the cells were placed in the upper chamber after transfection with RKIP, and the count was fixed after 24 hours (taking the average of 6 different regions), magnification, $\times 100$; (D) transwell invasion experiment: high expression of RKIP significantly inhibits the invasiveness of cells through the artificial basement membrane. RKIP, Raf-1 kinase inhibitor protein; NSCLC, non-small cell lung cancer. 


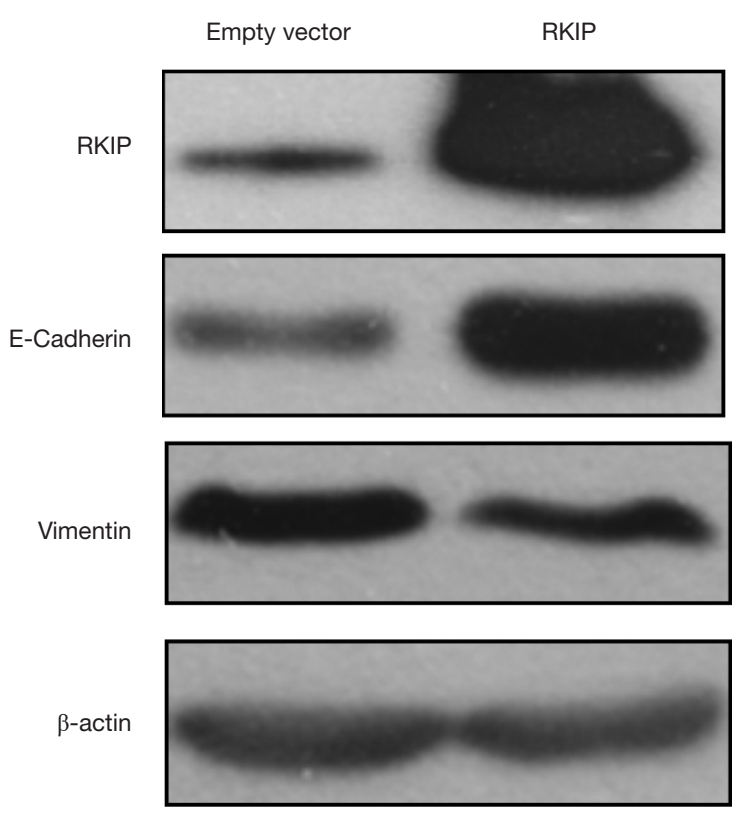

Figure 5 Overexpression of RKIP inhibits EMT in NSCLC cells. Western bolt analysis of protein level in A549 cells transfected with RKIP expressing plasmid or empty vector on EMT. RKIP, Raf-1 kinase inhibitor protein; EMT, epithelial-mesenchymal transition; NSCLC, non-small cell lung cancer.

tumors. This is not consistent with the results of this study. The occurrence and development of tumors is a very complex process, which is subject to a variety of cytokines in vivo. RKIP mainly affects the metastasis of cancer cells. Whether the expression of RKIP is related to tumor size in metastatic cancer remains to be confirmed by further study.

Tumor metastasis is a complex multi-step process, involving in situ invasion of tumor cells, angiogenesis, intravascular invasion, ability of tumor cells to survive in circulation, localization of cancer cells in the distance and proliferation. EMT is the first step in tumor metastasis. In addition to morphological changes, tumor cells activated by EMT secrete hydrolase to degrade extracellular matrix, forming pseudopodal processes anchored on specific ECM ligands, and migrate through cell contraction. In this process, the polarity of epithelial cells is lost, and the ability of movement and migration is enhanced. At the same time, mesenchymal phenotypes such as vimentin and fibronectin were gradually obtained by the loss of endophenotypes such as E-cadherin and keratin filaments. Current studies have found that growth factors, transcription factors, multiple kinases and signaling pathways can induce or participate in EMT.
The exact mechanism of RKIP inhibiting tumor metastasis is still not clear. Studies have shown that RKIP can affect the functions of MMP1, OPN and CXCR4 $(9,20)$. The main pathways involved are RafMEK-ERK and its downstream target c-myc (21). RKIP inhibits tumor metastasis by inhibiting angiogenesis, local invasion, intravascular invasion and proliferation. The molecular mechanism of each step is still unclear. RKIP reduction activates the Shh-Gli1 signaling pathway, thereby enhancing the expression of tumor stem cells (CSC) in NSCLC cell lines (13). In nasopharyngeal carcinoma cells, high expression of RKIP decreased invasion, metastasis and EMT marker alternations of cells (22). In NSCLC, whether RKIP can affect tumor invasion and metastasis through EMT is not reported in literature.

NSCLC cell lines involved in the study were used to verify the effect of RKIP on invasion and migration of lung cancer cells. It was found that the invasion and migration of lung cancer cells transfected with RKIP were significantly weakened, which was consistent with the clinical results. In this study, the relationship between RKIP and EMT was further explored in lung cancer. Experiments revealed that the expression of e-cadherin, a molecular marker of epithelial cells, was significantly increased after RKIP transfection, while the expression of the molecular marker vimentin of mesenchymal cells was significantly reduced, suggesting that RKIP regulates the invasion, metastasis and EMT of lung cancer cells.

Different from previous studies, we successfully transfected RKIP gene into A549 cells by plasmid. After overexpressing RKIP, the proliferation, activity, invasion, migration and movement of A549 cells were inhibited. Further studies also found that the main mechanism of RKIP inhibiting tumor invasion and metastasis was inhibition of EMT. This study revealed for the first time that RKIP inhibits tumor metastasis in lung cancer and is associated with EMT. Although the key signaling pathway of RKIP acting on EMT in NSCLC cells remains to be further studied. RKIP may be an important target for antitumor metastasis therapy.

\section{Conclusions}

In summary, this study not only found that RKIP was involved in the invasion and migration of lung cancer cells, but also blocked EMT by limiting tumor cells. The function and mechanism of RKIP as a tumor metastasis suppressor gene were fully verified in lung cancer cell lines. 
RKIP may be related to the occurrence and development of tumors.

\section{Acknowledgments}

Funding: This work was supported by grants from the National Natural Science Foundation of China (grant number 81401903, 81572937 and 81572273); the16th batch "Summit of the Six Top Talents" Program of Jiangsu Province (grant number WSN-154); China Postdoctoral Science Foundation 12th batch Special fund (Postdoctoral number: 45786); China Postdoctoral Science Foundation 64th batch (Postdoctoral number: 45786); Jiangsu Provincial Postdoctoral Science Foundation (grant number 2018K049A); the Natural Science Foundation of Jiangsu province (grant number BK20180139 and BK20161386); Jiangsu Provincial Medical Youth Talent (grant number QNRC2016125), and the Nanjing Medical Science and Technology Development Project (No. ZKX17044), the Jiangsu Provincial Key Research and Development Program (No. BE2016721).

\section{Footnote}

Conflicts of Interest: The authors have completed the ICMJE uniform disclosure form (available at http://dx.doi. org/10.21037/tcr.2019.09.56). The authors have no conflicts of interest to declare.

Ethical Statement: The authors are accountable for all aspects of the work in ensuring that questions related to the accuracy or integrity of any part of the work are appropriately investigated and resolved. The study was conducted in accordance with the Declaration of Helsinki (as revised in 2013). The study was approved by the Institutional Review Board (IRB) in Jinling Hospital (No. 2012NZJLS-220), and informed consent was obtained from all patients before present study initiated.

Open Access Statement: This is an Open Access article distributed in accordance with the Creative Commons Attribution-NonCommercial-NoDerivs 4.0 International License (CC BY-NC-ND 4.0), which permits the noncommercial replication and distribution of the article with the strict proviso that no changes or edits are made and the original work is properly cited (including links to both the formal publication through the relevant DOI and the license). See: https://creativecommons.org/licenses/by-nc-nd/4.0/.

\section{References}

1. Bray F, Ferlay J, Soerjomataram I, et al. Global cancer statistics 2018: GLOBOCAN estimates of incidence and mortality worldwide for 36 cancers in 185 countries. CA Cancer J Clin 2018;68:394-424.

2. Ettinger DS, Wood DE, Aisner DL, et al. Non-Small Cell Lung Cancer, Version 5.2017, NCCN Clinical Practice Guidelines in Oncology. J Natl Compr Canc Netw 2017;15:504-35.

3. Schmidt-Kittler O, Ragg T, Daskalakis A, et al. From latent disseminated cells to overt metastasis: Genetic analysis of systemic breast cancer progression. Proc Natl Acad Sci U S A 2003;100:7737-42.

4. Yang $\mathrm{MH}, \mathrm{Wu} \mathrm{MZ}$, Chiou SH, et al. Direct regulation of TWIST by HIF-1alpha promotes metastasis. Nat Cell Biol 2008;10:295-305.

5. Wu Y, Liu HB, Ding M, et al. The impact of E-cadherin expression on non-small cell lung cancer survival: a metaanalysis. Mol Biol Rep 2012;39:9621-8.

6. Yeung K, Seitz T, Li S, et al. Suppression of Raf-1 kinase activity and MAP kinase signalling by RKIP. Nature 1999;401:173-7.

7. Tang H, Park S, Sun SC, et al. RKIP inhibits NFkappaB in cancer cells by regulating upstream signaling components of the IkappaB kinase complex. FEBS Lett 2010;584:662-8.

8. Jia B, Liu H, Kong Q, et al. RKIP expression associated with gastric cancer cell invasion and metastasis. Tumour Biol 2012;33:919-25.

9. Yun J, Frankenberger CA, Kuo WL, et al. Signalling pathway for RKIP and Let-7 regulates and predicts metastatic breast cancer. EMBO J 2011;30:4500-14.

10. Martinho O, Pinto F, Granja S, et al. RKIP inhibition in cervical cancer is associated with higher tumor aggressive behavior and resistance to cisplatin therapy. Plos One 2013;8:e59104.

11. Wang $\mathrm{Q}, \mathrm{Wu} \mathrm{X}, \mathrm{Wu} \mathrm{T}$, et al. Clinical significance of RKIP mRNA expression in non-small cell lung cancer. Tumour Biol 2014;35:4377-80.

12. Wang A, Duan G, Zhao C, et al. Reduced RKIP expression levels are associated with frequent non-small cell lung cancer metastasis and STAT3 phosphorylation and activation. Oncol Lett 2017;13:3039-45.

13. Xie SY, Li G, Han C, et al. RKIP reduction enhances radioresistance by activating the Shh signaling pathway in non-small-cell lung cancer. Onco Targets Ther 2017;10:5605-19. 
14. Beach S, Tang H, Park S, et al. Snail is a repressor of RKIP transcription in metastatic prostate cancer cells. Oncogene 2008;27:2243-8.

15. Bollengier F, Mahler A. Localization of the novel neuropolypeptide h3 in subsets of tissues from different species. J Neurochem 1988;50:1210-4.

16. Seddiqi N, Bollengier F, Alliel PM, et al. Amino acid sequence of the Homo sapiens brain 21-23-kDa protein (neuropolypeptide h3), comparison with its counterparts from Rattus norvegicus and Bos taurus species, and expression of its mRNA in different tissues. J Mol Evol 1994;39:655-60.

17. Frayne J, Ingram C, Love S, et al. Localisation of phosphatidylethanolamine-binding protein in the brain and other tissues of the rat. Cell Tissue Res 1999;298:415-23.

18. Vallée BS, Tauc P, Brochon JC, et al. Behaviour of bovine phosphatidylethanolamine-binding protein with model membranes. Evidence of affinity for negatively charged

Cite this article as: Wang Q, Li XY, Wan B, Zhang J, Sun R, Zhou CY, Zhan P, Song Y. Overexpression of Raf-1 kinase inhibitor protein inhibits cell invasion and migration in lung cancer cells through suppressing epithelial-mesenchymal transition. Transl Cancer Res 2019;8(6):2295-2306. doi: 10.21037/ tcr.2019.09.56 membranes. Eur J Biochem 2001;268:5831-41.

19. Reumer A, Bogaerts A, Van Loy T, et al.

Unraveling the protective effect of a Drosophila phosphatidylethanolamine-binding protein upon bacterial infection by means of proteomics. Dev Comp Immunol 2009;33:1186-95.

20. Beshir AB,Ren G,Magpusao AN, et al. Raf kinase inhibitor protein suppresses nuclear factor-kB-dependent cancer cell invasion at the level of matrix metalloproteinase expression but not cell migration. Cancer Lett 2010;299:137-49.

21. Tsao DA, Tseng WC, Chang HR. The expression of RKIP, RhoGDI, galectin, c-Myc and 553 in gastrointestinal system of $\mathrm{Cr}(\mathrm{VI})$-exposed rats. J Appl Toxicol 2011;31:730-40.

22. He QY, Yi HM, Yi H, et al. Reduction of RKIP expression promotes nasopharyngeal carcinoma invasion and metastasis by activating Stat 3 signaling. Oncotarget 2015;6:16422-36. 